\title{
The Transillumination Possibility of Imidazole-Osmium Postfixed Muscle Tissue and Its Consequences for the Handling of Muscle Tissue Samples
}

\author{
TILMAN VOIGT ${ }^{1 *}$ AND WOLFGANG DAUBER ${ }^{2}$ \\ ${ }^{1}$ Institute of Anatomy, University of Fribourg, CH-1700 Fribourg, Switzerland \\ ${ }^{2}$ Institute of Anatomy, Eberhard-Karls-University Tuebingen, D-72074 Tuebingen, Germany
}

KEY WORDS imidazole; osmium; fixation; technique; electron microscopy; light microscopy; skeletal muscle

\begin{abstract}
The osmium postfixation of tissue leads to good results for transmission electron microscopy, but also produces completely blackened tissue samples that do not allow the recognition of internal structures. With imidazole-osmium postfixation, one achieves comparable results in high electron microscopic resolution as with routine osmium postfixation. But the tissue samples are not blackened and can, therefore, be transilluminated with point light sources. The new postfixation technique makes it possible to recognize histological details such as vessels, nerve fibers, and the cross-banding pattern in an untrimmed block. This makes it possible to screenembedded tissue samples for appropriate ultrastructural processing.
\end{abstract}

\section{INTRODUCTION}

Osmium-postfixation is established as a routine method for tissue preparation for transmission electron microscopy (TEM). A disadvantage of the osmium postfixation is, however, that through the osmium the tissue samples are completely blackened. This means that structures cannot be discerned within the tissue samples. Questions as to whether and where a certain structure is available within the tissue samples cannot be addressed after osmium postfixation. Usually this problem is solved by the cutting and examining of semithin sections. In our investigations of the motor end plate, we became aware of imidazole-osmium postfixation, which supplies comparable postfixation results for high electron microscopic resolution (Voigt et al., 2003). During postfixation, the tissue samples turn reddish in color and they can be transilluminated with point light sources. In the present work, we use muscle tissues of frog and mouse to illustrate the consequences this form of transillumination could have for the handling of embedded tissue samples.

\section{MATERIALS AND METHODS}

The investigations were carried on the diaphragm of mice and the Musculus sartorius of Rana temporaria.

Following anaesthesia with a mixture of Rompun (Bayer), and Ketanest (Parke, Davis \& Company), perfusion of the mice was performed from the left ventricle by pre-rinsing with Thomaedex 40 (Thomae) and fixation with $2.5 \%$ glutaraldehyde in $0.1 \mathrm{M}$ cacodylate buffer. The diaphragm was cut out with the rib frame and this dissect was fixed in the same fixing solution for 1 hour. Subsequently, muscle samples were cut to size and fixed in $2.5 \%$ glutaraldehyde for 2 hours.

The frogs were anaesthetized with MS222 (Sandoz Co.) and decapitated. The legs were exarticulated at the pelvic joint. In a Petri dish filled with $2.5 \%$ glutar- aldehyde in $0.1 \mathrm{M}$ cacodylate buffer, the skin of the leg was removed, the superficial Musculus sartorius exposed, and its fascia completely removed. Then the entire leg was immersion-fixed in the same fixative for 1 hour. Subsequently, the muscle was divided into small strips of tissue and allowed to remain in the fixative agent for a further 2 hours.

The tissue samples of mice and frog were divided into two groups and subjected in parallel to post-fixation for 1 hour in (1) $1 \%$ osmium in $0.1 \mathrm{M}$ cacodylate buffer or (2) in $1 \%$ osmium with $0.1 \mathrm{M}$ imidazole in $0.1 \mathrm{M}$ cacodylate buffer.

Following this, all of the samples were washed for $3 \times 5 \mathrm{~min}$ in Aqua. dest. and then dehydrated in an ascending alcohol series and embedded in EPON so that several EPON-blocks are obtained.

It is to be noted that if a label is placed in the EPON-block, this does not disturb the beam path through the embedded tissue sample.

The tissue samples embedded in the EPON-blocks were then analyzed under a binocular (Wild Heerbrugg Photomakroskop M400) or a light microscope (Zeiss, Oberkochen, Germany) both in the untrimmed and trimmed state. In the trimmed state, the surface of the EPON-block is polished by the cutting of semithin sections. But the embedded tissue sample does not need to be cut when searching for structures within the tissue sample. It is only the surface of the EPON-block that is

\footnotetext{
*Correspondence to: Tilman Voigt, Institute of Anatomy, University of Fribourg, Rte Albert-Gockel 1, CH-1700 Fribourg, Switzerland. E-mail: tilman voigt-gawatz@unifr.ch

Received 11 July 2003; accepted in revised from 16 October 2003

The primary institution for this research is the Institute of Anatomy, University of Fribourg, CH-1700 Fribourg, Switzerland.

DOI 10.1002/jemt.20021

Published online in Wiley InterScience (www.interscience.wiley.com).
} 


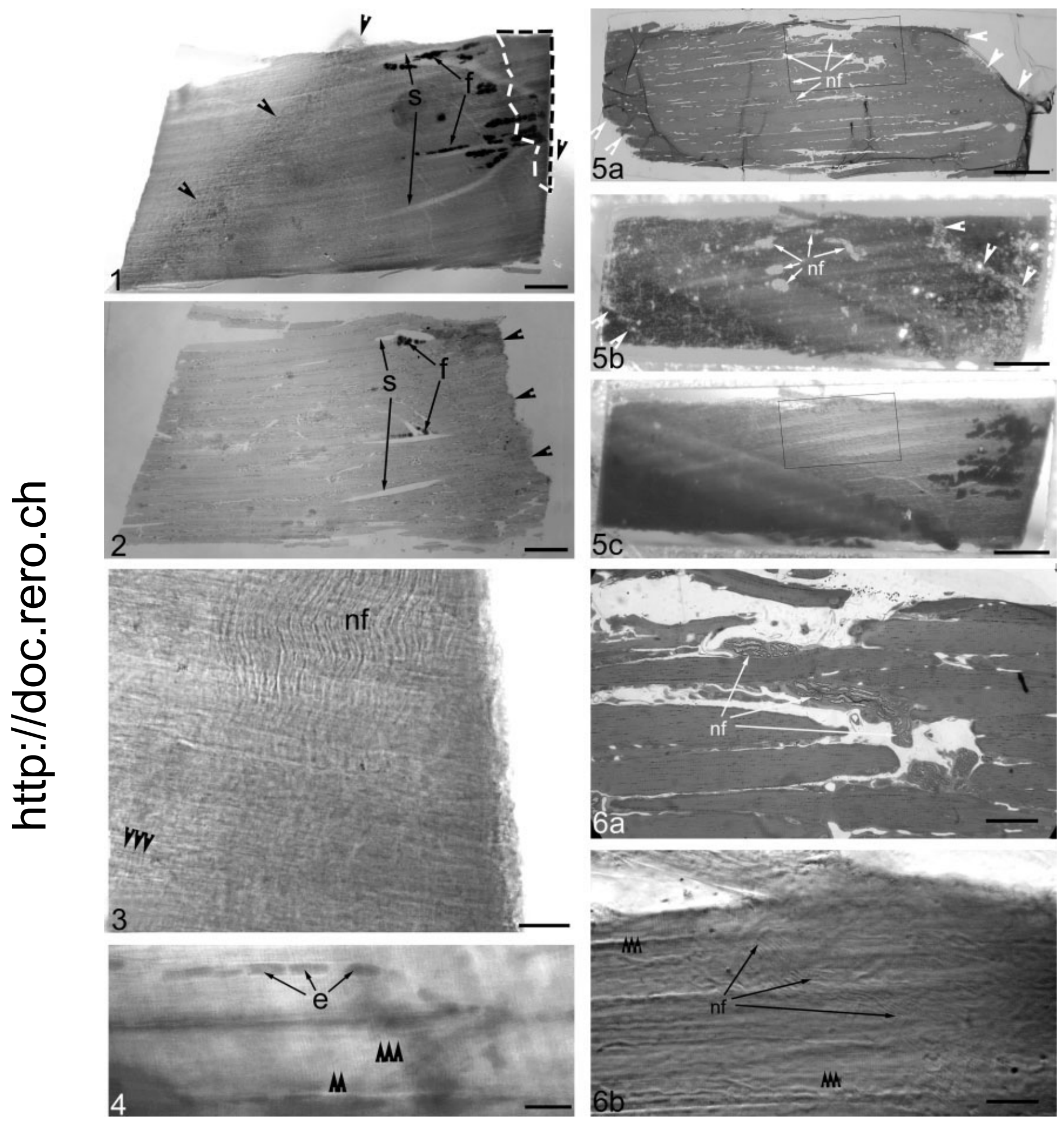

Figs. 1-6 
decisive for the reproduction quality in the beam path of the optical microscope. The embedded tissue samples in the EPON-blocks were photographed via the ocular with a digital camera (Canon PowerShot S40, aperture 8 , exposure time 1'; light-sensitivity ISO50, ocular adapter, Leica). Semithin sections were stained according to Richardson et al. (1960).

\section{RESULTS}

During imidazole-osmium postfixation, the tissue samples turn reddish. A reddish precipitate that sometimes forms does not disturb the result of the postfixation. After embedding in EPON, the imidazole-osmium postfixed tissue samples remain reddish.

With point light sources, the imidazole-osmium postfixed diaphragm of the mouse (about $200 \mu \mathrm{m}$ thick) and the Musculus sartorius of Rana temporaria (about $500 \mu \mathrm{m}$ thick) can be transilluminated (Figs. 1, 4). In spite of identical thickness, the osmium postfixed diaphragm can no longer be transilluminated (not shown).

After imidazole-osmium postfixation, one can recognize fat cells (Fig. 1) and melanocytes (not shown) between muscle fibers in untrimmed EPON-blocks. In untrimmed EPON-blocks, branched-out structures in the embedded muscle tissue can be recognized as vessels if, as with the unperfused frog muscles, they are filled with erythrocytes (Fig. 4). Also the cross banding of skeletal muscle fibers, can be recognized in the untrimmed EPON-blocks (Fig. 1, 4). The rough surface of the untrimmed EPON-block leads, however, to optical interference in the reproduction quality. Vessels in perfused muscles of mice are also seen as branched-out structures in the transilluminated EPON-blocks (Fig. 1). Because after perfusion these structures are not filled with erythrocytes, they only can be identified as vessels in the semithin section (Fig. 2).

Through the cutting of sections, the surface of the EPON-block is polished and, therefore, the optical resolution is improved. Then, for example, also the course of nerve fibers in the embedded tissue sample can be shown in the light microscope (Fig. 3). The nerve fiber bundle can be recognized in the embedded muscle tissue, long before these nerve fibers appear in the semithin section (Fig. 3).

To prove that the wavy structure that we show in Figure 3 is nerve fibers, we show in Figures 5 and 6 a nerve that can be co-localized in the semithin section and the transilluminated block. For this purpose, a block was sectioned as far as a nerve could be followed in the semithin section on a maximally long route (Figs. 5a, 6a). For precise localization of the nerve fibers, the semithin section (Figs. 5a, 6a), the reflections of the block surface (Fig. 5b) and the transilluminated block (Figs. 5c, 6b) were photographed. By means of landmarks (border of embedded tissue; border of semithin section; reflection image of sectioned nerves), it can be shown that the wavy structure seen by transillumination (Figs. 5c, 6b) co-localize with the nerve fibers in the semithin section (Figs. 5a, 6a).

\section{DISCUSSION}

Imidazole-osmium postfixation is a method that can be applied easily by addition of a corresponding quantity of imidazole to the osmium. The advantages of the imidazole-osmium postfixation for investigations at the TEM have been described elsewhere (Angermüller and Fahimi, 1982; Goldberg and Septier, 1984; Thiéry et al., 1995; Voigt et al., 2002, 2003). On the one hand, lipid-containing structures (membranes, lipid-droplets) are better stained by this postfixation (Angermüller and Fahimi, 1982; Goldberg and Septier, 1984; Thiéry et al., 1995; Voigt et al., 2002). On the other hand, the investigation of thick sections in the TEM is facilitated by the improvement in contrast (Voigt et al., 2003). Finally, the still unstained section can be analyzed natively in the TEM, so that much work can be saved in the processing of the ultrathin sections (Voigt et al., 2002). Since imidazole dissolves immediately in aque-

Fig. 1. Transilluminated untrimmed EPON-block of the flat embedded diaphragm of the perfused mouse; thickness of diaphragm approximately $200 \mu \mathrm{m}$. Aggregation of fat cells $(\mathrm{f} \rightarrow$ ) can be shown between the muscle fibers. Brighter structures run obliquely, longitudinally and across the muscle fibers $(\mathrm{S} \rightarrow$ ); shadow of the label that distorts the beam path through the tissue sample (arrowheads). In the semithin section (see Fig. 2), the enclosed field is not yet cut. Imidazole-osmium postfixation, bar $=250 \mu \mathrm{m}$.

Fig. 2. Semithin section of the EPON-block shown in Figure 1. In the section, the bright structures (s $\rightarrow$ ) recognized in the untrimmed EPON-block (see Fig. 1) turn out to be vessels. In the section, no nerve fibers can be shown. Yet the embedded tissue is not sectioned completely; compare the right top border (arrowheads) of the tissue in the semithin section with the corresponding enclosed field of the embedded tissue sample (see Fig. 1); fat cells (f). Imidazole-osmium postfixation, bar $=250 \mu \mathrm{m}$.

Fig. 3. Detail of the transilluminated trimmed EPON-block after semithin section of Figure 2 in the range of the enclosed field of the embedded tissue sample (see Fig. 1). In the muscle tissue sample, the course of nerve fibers (nf) as well as the cross-banding pattern of muscle fibers can be shown (arrowheads). Because of the small enlargement, only every second cross-band is labeled by the arrowheads. Trimmed EPON-block with polished surface; imidazole-osmium postfixation, bar $=25 \mu \mathrm{m}$.

Fig. 4. Transilluminated untrimmed EPON-block of the unperfused Musculus sartorius of the frog; thickness of muscle sample approximately $500 \mu \mathrm{m}$. Vessels, which are filled with erythrocytes $(\mathrm{e} \rightarrow)$, run between muscle fibers. The cross-banding pattern of muscle fibers can be shown (arrowheads). Because of small enlargement, only every second cross-band is labeled by the arrowheads. Imidazole-osmium postfixation, bar $=30 \mu \mathrm{m}$

Fig. 5. Figures of an embedded imidazole-osmium postfixed muscle tissue sample after the search for nerve fibers. a: semithin section; b: reflection image of the surface of the EPON-block; c: transillumination of the EPON-block. In the semithin section (a) nerve fibers (nf $\rightarrow$ ) can be shown, which can be recognized also in the reflection image (b). The outline of the embedded tissue sample can be recognized both in the reflection image (b) and after transillumination (c). In the reflection image (b), the border (arrowheads) of the semithin section (a) can be traced; yet the embedded tissue is not sectioned completely. Details in Figure 6 correspond to the black frame in the semithin section (a) and in the transillumination (c). Trimmed EPON-block with polished surface; imidazole-osmium postfixation, bar $=250 \mu \mathrm{m}$.

Fig. 6. a: Framed detail of Figure 5a. In the semithin section, nerve fibers $(\mathrm{nf} \rightarrow$ ) run across and obliquely between muscle fibers. Trimmed EPON-block with polished surface; imidazole-osmium postfixation, bar $=50 \mu \mathrm{m}$. b: Framed detail of Figure $5 \mathrm{c}$. The same nerve fibers (nf $\rightarrow$ ), shown at the semithin section (a) can be recognized by transillumination of the embedded tissue sample. The cross-banding pattern of the muscle fibers can be shown (arrowheads). Due to the small enlargement, only every second cross-band is labeled by arrowheads. Trimmed EPON-block with polished surface; imidazole-osmium postfixation, bar $=50 \mu \mathrm{m}$. 
ous solutions, there are no complications in the preparation of the solution. The weakly yellowish osmiumsolution becomes reddish through the addition of imidazole. In our experience, sometimes a reddish precipitate forms during postfixation, but this does not have any influence on the quality of the postfixation.

Since the tissue samples are blackened completely during osmium postfixation, a search for structures of interest within the postfixed embedded tissue sample is impossible. After imidazole-osmium postfixation, the tissue samples are only reddish and can be transilluminated. In the handling of embedded tissue samples, including section for the TEM, the possibility of the transillumination of embedded tissue samples in the EPON-block can be a further advantage. Because of the transillumination possibility, some structures, like blood vessels, nerve fibers, and finer details such as the cross-banding pattern of skeletal muscle fibers, can be recognized in both untrimmed and trimmed EPON-blocks.

The resolution in the translucent beam path of the light microscope is dependent on the thickness of the embedded tissue samples, and on the existence of certain foreign bodies in the EPON-block (i.e., labels) and the surface of the EPON-block. But in spite of distortion of the beam path by the label in our EPON-block, the above-mentioned structures could be shown.
In the search for motor end plates, knowledge of the existence and location of nerve fibers in an embedded tissue sample or in EPON-blocks is of great advantage, since motor end plates can mostly be found in their proximity. Thus, a preselection of EPON-blocks can be carried out after the imidazole-osmium postfixation. In addition, the structures recognized in the embedded tissue sample can be useful for orientation in the section during investigation with the TEM.

\section{REFERENCES}

Angermüller S, Fahimi HD. 1982. Imidazole-buffered osmium tetroxide: an excellent stain for visualization of lipids in transmission electron microscopy. Histochem J 14:823-835.

Goldberg M, Septier D. 1984. Demonstration of lipids by imidazolebuffered osmium tetroxide in the odontoblasts and cells of the enamel organ in the rat incisor. J Biol Buccale 12:317-330.

Richardson KC, Jarett L, Finke EH. 1960. Embedding in epoxy resins for ultrathin sectioning in electron microscopy. Stain Technol 35: 313-323.

Thiéry G, Bernier J, Bergeron M. 1995. A simple technique for staining of cell membranes with imidazole and osmium tetroxide. J Histochem Cytochem 43:1079-1084.

Voigt T, Dauber W, Bensemann-Ryvkin I, Härtel X. 2002. Increasing membrane contrast by means of imidazole-osmium post-fixation as exemplified by skeletal muscle fiber. Microsc Res Tech 58:121-124.

Voigt T, Dauber W, Bensemann-Ryvkin I, Härtel X. 2003. The shape and the position of the sarcoplasmic reticulum and the Golgi apparatus in the sole plate and the remaining subsarcolemmal muscle region of the mouse using Imidazole-Osmium staining. Microsc Res Tech 61:419-422. 